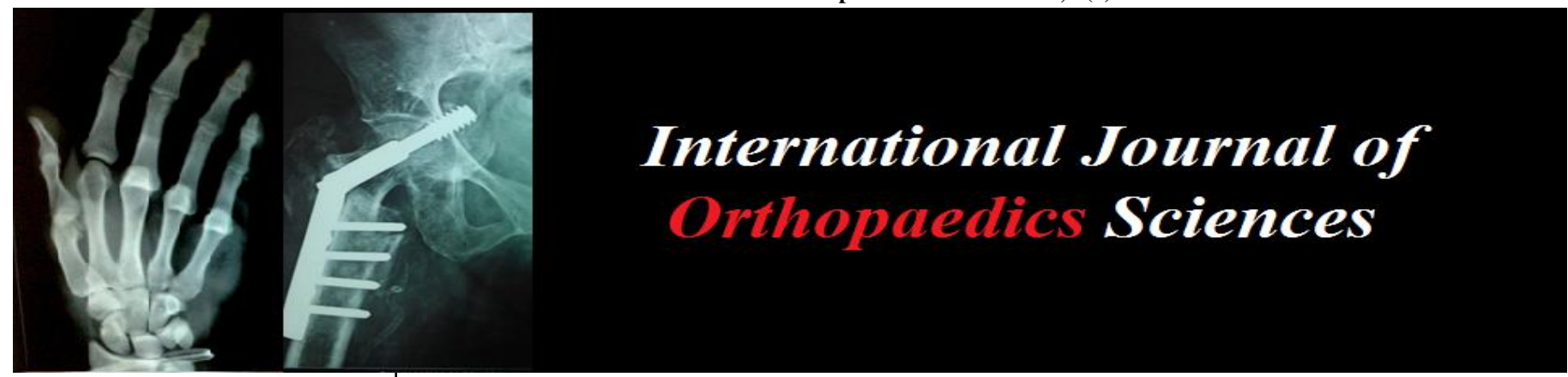

ISSN: $2395-1958$

IJOS 2019; 5(3): 298-303

(C) 2019 IJOS

www.orthopaper.com

Received: 21-05-2019

Accepted: 26-06-2019

Nixon Dias

Department of Orthopaedics, ST Martha's Hospital, Nrupathunga Road, Bangalore, Karnataka, India

\section{Jayanth Kumar}

Department of Orthopaedics, ST Martha's Hospital, Nrupathunga Road, Bangalore, Karnataka, India
Correspondence

Nixon Dias

Department of Orthopaedics, ST Martha's Hospital, Nrupathunga Road, Bangalore, Karnataka,

India

\section{A prospective study of the functional outcomes in operative management of intra-articular Calcaneum fractures}

\section{Nixon Dias and Jayanth Kumar}

DOI: https://doi.org/10.22271/ortho.2019.v5.i3e.1544

\section{Abstract}

Background \& Objectives: The treatment of intra-articular fractures of the calcaneum remains controversial. There is no consensus in the current literature regarding the optimal treatment of intraarticular fractures of the calcaneum. In the current prospective study, we intend to study the outcome of operative management for intra-articular calcaneum fractures based on the objective criteria, that is, restoration of Bohler's angle, subtalar range of motion and subjective criteria such as pain, return to work, return to physical activity, change in shoe wear.

Materials and Method Design

Prospective study: Study was conducted in St Martha's Hospital, Bangalore, during the period extending from Nov'2014 to Dec'2016. 15 patients with 17 intra-articular calcaneum fracture were included in the study. Outcome of operative management was evaluated using Modified Rowes scoring system. Pretreatment and post treatment (at follow up) Bohler's angle and Gissane's angle was also compared. Chi-square analysis and student ' $t$ ' test was done to analyze the results of operative management.

Results: Restoration of the Bohler's angle was achieved with operative management. Functional outcome for type I and II fractures was better than with type III and IV. A significant correlation was seen between the post-operative Bohler's angle and Rowe scores.

Interpretation \& Conclusion: Operative management has a good to fair functional outcome for displaced calcaneal fractures. For displaced and comminuted fractures anatomical reduction and restoration of Bohler's angle is very important. Bohler's angle has a prognostic importance and correlates well with the functional outcome.

Keywords: Intra-articular calcaneum fracture, operative, Rowe score, Bohler's angle

\section{Introduction}

The calcaneus (os calcis) is the largest and most often fractured tarsal bone in the foot. It is the major weight bearing osseous structure of the foot and is one of the components of the tritarsal articulation and has important functional tasks with regard to normal ambulation. Being so vital to the normal mechanics of locomotion, it is easy to see why a fracture of calcaneus is attended by considerable morbidity. Despite the Surgeons extensive experience with this injury, its major socio-economic impact in regard to the time lost from work, and the attention given to it for many years throughout the world, there is still no method of treatment that yields consistently good results in these injuries.

Bankart ${ }^{[1]} 35$ years later, wrote,' the results of treatment of crush fractures of heel are rotten"... the best result that can be expected from a fracture of the subastragloid joint is a completely stiff but painless foot of a good shape with free movement of the entitled joint.

Conn ${ }^{[2]}$ in 1932 wrote that fractures of calcaneus are "serious and disabling injuries in which the end results continue to be incurably bad". Bohler ${ }^{[3]}$ began to advocate open reduction of calcaneal fractures in 1931 based on his experience with the French methods. Despite this, forcible closed reduction with tongs and hammers or traction followed by manual manipulation and casting, were the standard treatments of his time, because of technical problems associated with surgery. During the 1950s, varied techniques were employed, but because subtalar fusion was the easiest to perform, it became the most commonly practiced treatment. 
Over the last 25 years, however, marked advances in anesthesia, prophylactic antibiotics, computed tomography (CT) scanning, and fluoroscopy have allowed surgeons improve outcomes when operating on fractures and these techniques have been applied to calcaneal fractures as well ${ }^{[4]}$. Overall, operative treatment of acute fractures has become the standard of care with many surgeons who have critically evaluated their results and concluded that good outcomes are possible.

Despite these improvements, it is recognized that operative treatment is still beset with difficulties.

\section{Material and Methods}

This study was done to evaluate the results of operative management for intra-articular calcaneum fractures on basis of subjective, clinical and radiological criteria in our hospital setup.

2.1 Study design: This is a prospective evaluation.

2.2 Sample size: Hospital records for the past three years show an average of 15-25 patients meeting the criteria.

Rule of thumb calculation of sample size (per variable minimum 5 subjects and maximum 10 subjects) show sample size of 15-25 Sample size estimation could then be estimated after doing pilot study. So, the sample size is going to be 15 patients.

\subsection{Inclusion criteria}

a. Patients aged 18 years or more will be included in the study

b. Patients with a displaced intra-articular calcaneal fracture undergoing surgical fixation in our hospital.

c. Patients with a calcaneal fracture of the Sanders types I, II, III or IV, undergoing surgical fixation in our hospital.

\subsection{Exclusion criteria}

a. Patients with pathological fractures

b. Patient having old or neglected fractures, or those treated after more than 14 days of occurrence of fracture

c. Patient with malunited fractures of the ankle, with associated complications like subtalar arthritis

d. Patients with a severely comminuted calcaneal fracture.

e. Patients with multiple injuries; such as those having an associated spine and cord injury.

2.5 Period of study: $1^{\text {st }}$ October 2014 to $31^{\text {st }}$ December 2016 . Follow up period was for 1 year.

2.6 Study area and population: It is a hospital based prospective study involving the out-patient department, ward and the operation theatre of St. Martha's Hospital for obtaining the data. Study population is the patients coming to our hospital with calcaneal fractures.

2.7 Method of data collection and procedure involved in the study: When the patients were seen for the first time after injury, a thorough history was taken regarding the time of injury, mechanism of injury, any significant past or personal history (especially diabetes and smoking) and was recorded. Patients were examined giving special importance to whether the fracture was open or closed, presence of gross swelling, fracture blisters, features of compartment syndrome and presence of other associated injuries. Routine investigations were done as were necessary. The diagnosis was confirmed by antero-posterior (dorsoplantar), lateral and axial radiographs. CT scan was done to assess the fracture pattern, displacement of fracture fragments, comminution and to classify the fracture according to Sanders classification. Bohler's angle was measured on X ray. Open fractures were classified according to Gustilo Anderson classification.

All the cases were treated as in-patients. The form of treatment, conservative or operative was decided depending on type of fracture, patients age, condition of soft tissues, associated injuries, co-morbidities, patients occupation, affordability as well as surgeon's decision. The patients who underwent operative management were evaluated in this study.

All the patients were found to have swelling, which was subsequently treated with limb elevation, local ice packs and anti-inflammatory drugs and a below knee POP slab. All the patients were advised NWB walking for 12 weeks.

The main indication for operative management was displaced intra-articular fractures with incongruous subtalar joint and reduced Bohler's angle. The method of reduction and fixation were decided according to the fracture pattern, the surgeons experience, associated injuries and affordability of the patient. Closed reduction with or without fixation was done within 2448 hours of injury and open reduction and fixation was done once the skin condition was good but within 3 weeks of injury. The basic idea was to achieve near anatomical reduction and a congruous subtalar joint.

Essex-Lopresti reduction was done in 12 cases. With patients in prone or supine position with external rotation and foot in lateral position for ease of manipulation and imaging under $\mathrm{C}$ arm guidance, a $3 \mathrm{~mm} \mathrm{~K}$ wire was inserted through the posterior end of calcaneus into the tongue fragment. This fragment was levered into reduced position using the reduction manoeuvre. Then the $\mathrm{K}$ wire was driven into anterior process. 2 cannulated cancellous 6.5 screws were inserted over the guide wires, each medial and lateral to the Achilles insertion. Additional $4.5 \mathrm{~mm}$ screws were inserted into the sustentacular fragment if and when required. A below knee cast was applied following this.

Closed reduction and $\mathrm{K}$ wire fixation was done for 1 patient, with an open wound posteriorly. In this after achieving the reduction and maintaining the Bohler's angle, the reduction was secured with $\mathrm{K}$ wires. B K slab was put following this. At end of 2 weeks, as the swelling subsided, the wires were removed and $6.5 \mathrm{~mm}$ cannulated cancellous screws were inserted in an appropriate fashion and compression achieved. The patient underwent skin grafting for the raw area and exposed Achilles tendon following this. Slab was applied and patient maintained NWB.

Open reduction and internal fixation was used in 4 patients. It was done as a planned and elective procedure. Before patients were taken up for surgery, limb elevation, local ice packs, glycerine magsulf dressing and anti-inflammatory drugs was advised for few days to reduce the foot swelling. For all 4 cases Seligson's lateral approach was used.

The fracture was reduced and temporarily fixed with $\mathrm{K}$ wires, if needed. The definitive fixation was done with help of special calcaneum plates in 3 cases and reconstruction plate in 1 case. Wound was closed in the standard fashion with a vacuum drain. Post operatively limbs were immobilized in plaster cast, patients were put on antibiotics and analgesics and limbs were elevated. Wounds were inspected on third post-operative day by creating a window over the wound in the cast and sutures were removed (on average) on twelfth day (decided according to the wound condition). Once the wound healed well, window was closed and cast reinforced. 
Patients were advised strict non-weight with axillary crutches. Cast was removed at 6 weeks and fracture was assessed radiologically for union. Physiotherapy exercises for ankle and sub-talar movements were started on removal of cast. Once the fracture united radiologically, patients were started on partial weight bearing walking which was gradually increased to full weight bearing.

There were 4 open calcaneum fractures. 1 of which was a type IIIA with impending compartment syndrome of the foot which was managed on an emergency basis with temporary $\mathrm{K}$ wires and wound debridement along with fasciotomy. Another 1 of these open fractures, a Sanders type 2 fracture, was managed with percutaneous axial CC screws after debridement and wound closure. The other 2 open fractures were managed with plate fixation on the lateral surface following a type 2 wound closure on the medial surface. All these patients were kept on a POP slab post operatively for 6 weeks, and regular dressings of the wound were done. Non weight bearing mobilization was done and sutures were removed on the $12^{\text {th }}$ post-operative day in accordance with the wound status.

\subsection{Follow up}

For patients diagnosed as having calcaneum fracture, follow up was done every 2 weeks, 6 weeks, 3 months and then at 6 months since the time of treatment for clinical and radiological evaluation. Final evaluation was done at 12 months post surgery and functional outcome was scored.

They were also examined for presence of any residual swelling, deformity, condition of wound, tenderness and subtalar range of movements.

During follow up visit patients were asked regarding any complaints of pain, any, difficulty in walking (especially on uneven ground), change in daily activities and change in occupation. Patients were examined for any gait abnormalities (like antalgic gait or painless limping), weakness of triceps surae and any neurological deficits in foot (Eg: sensory loss over lateral border of foot in operated cases).

Follow up X-rays were taken to assess fracture union at every follow up visit, the condition of implant, to look for subtalar arthritis and any deformities. Bohler's angle was also calculated at this time and used to compare with pretreatment angle.

\subsection{Assessment of results}

Rowe scoring system was used to evaluate the patients. Pretreatment and follow up Bohler's angle and Gissane's angle was also compared using student " $t$ " test. Finally correlation between Rowe score and mean Bohler's angle and Gissane's angle was evaluated.

\subsection{Method of Statistical Analysis}

The student ' $\mathrm{t}$ ' test $\mathrm{t}^{5}$ was used to determine whether there was a statistical difference between Group 1 and Group 2 in the parameters measured.

In all the above test a "p" value of less than 0.05 was accepted as indicating statistical significance.

\section{Results}

A total of 15 patients with 17 fractures were enrolled in the study. All our patient were above 18 years of age. 2 cases had bilateral fractures without associated spinal fractures and both sustained by a fall from height. 12 cases of intra-articular fractures were managed with percutaneous screw fixation, among which 1 was an open fracture. 4 cases were managed with plate fixation, among which 2 were closed and 2 were open fractures. 1 compound fracture was managed with $\mathrm{K}$ wire stabilization and later conversion to screws.

According to Sanders classification of calcaneal fractures, the most common type seen in this study was that of Sander type $3,52.9 \%$ of the cases.

Majority of the patients, although, had no co-morbidities. $11.8 \%$ of the patients had diabetes, and these patients developed wound complications. Multiple trials earlier have reported similar observations.

Table 1: Pre and Post-Operative Bohlers Angle

\begin{tabular}{|c|c|c|c|c|c|}
\hline & $\mathbf{N}$ & Minimum & Maximum & Mean & Std. Deviation \\
\hline Pre BA & 17 & 4 & 15 & 9.35 & 3.181 \\
\hline Post BA & 17 & 22 & 32 & 26.18 & 2.628 \\
\hline
\end{tabular}

As shown above, it is seen that the mean pre-operative Bohler's angle was found to be $9.35^{\circ}$. The mean postoperative Bohler's angle was found to be $26.18^{0}$.

On measuring the pre and post-operative Gissane's angle in the cases, the mean pre-operative Gissanes angle was found to be $157.65^{\circ}$ while the mean post-operative Gissanes angle was found to be $132.35^{\circ}$.

As per the study by Yeo et al. ${ }^{[6]}$, median Bohler angle had improved to $26.5^{0}$ at the final follow up; median Gissanes angle had improved to $115.5^{\circ}$ at final follow up.

In our study, majority of the patients had a good functional outcome, $41.17 \%$ of patients. $23.52 \%$ of patients had an excellent outcome while another $23.52 \%$ had a fair outcome. $11.76 \%$ of the patients had a poor outcome as per Rowe score due to complaints of pain while walking. On comparison with earlier studies; as according to $\mathrm{Su}$ et al. ${ }^{[7]}$, who used American Orthopaedic Foot \& Ankle Society hindfoot score, the functional outcomes were excellent in $37.96 \%$ ), good in $48.18 \%$, fair in $9.85 \%$, and poor in $4.01 \%$.

The commonest complication found was stiffness/pain of the subtalar joint motion which was evidence of subtalar arthrosis; $23.52 \%$ patients had variable amounts of stiffness and thereby difficulty in walking on uneven surfaces. Another common complication seen was skin necrosis of the operative wound; seen in $17.6 \%$ of patients. A similar result was also found in the study conducted by Zhang et al. ${ }^{[8]}$, which had $15 \%$ of infectious complications in their study.

It was seen that the absence of co-morbidities in the patients resulted in a better mean function outcome. The presence of any of the co-morbidities, as listed previously resulted in a decreased end functional outcome. Diabetes was the strongest risk factor for wound complications ${ }^{[8]}$.

Table 2: Correlation between Post-Operative Bohlers Angle and Functional Outcome

\begin{tabular}{|l|c|c|c|c|}
\hline & & & Post BA & 12 months \\
\hline \multirow{3}{*}{ Spearman's } & \multirow{3}{*}{ Post BA } & Correlation Coefficient & 1.000 & $.693^{* *}$ \\
\cline { 3 - 5 } & & $\mathrm{p}$ value &. & .002 \\
\cline { 3 - 5 } & $\mathrm{N}$ & 17 & 17 \\
\hline
\end{tabular}

This table shows the positive correlation between post-operative Bohler's angle and the end functional outcome.

The Spearman's Correlation Coefficient is 0.693 and p value is 0.002 . This is evidence that, the improved Bohler's angle attained post operatively led to improved functional outcomes. Similar study was done by D Makki et al. [9] concluded that Restoration of Bohler's angle was associated with a better outcome and that prompt osteosynthesis should be considered for intra-articular fractures of the calcaneum in 
order to restore the shape of the hindfoot and Bohler's angle.

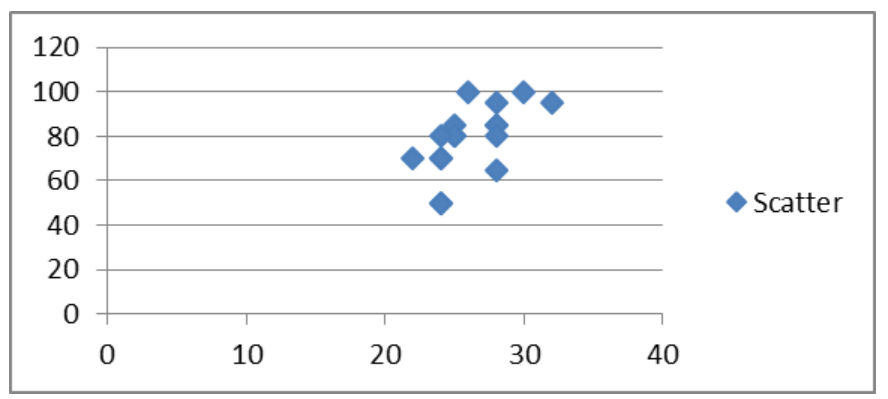

Fig 1: Scatter Plot of Post Op Bohlers with Functional Outcome

As seen in the Scatter plot above, that depicts the postoperative Bohler's angles and the end functional outcomes of these patients, it is seen that there was better functional outcomes in patients when they had improved Bohler's angles post operatively.

It was evidently noted that the patients who developed no complications had a better functional outcome than those patients who had complications.

\section{Discussion}

Open reduction and internal fixation has been established as a standard therapy for calcaneal fractures with good to excellent results. However, as surgical techniques have advanced, there remains uncertainty regarding the complication following operative management of calcaneal fractures.

In this study the outcomes of operatively managed calcaneal fractures was evaluated in order to find out the functional outcomes at the end of 1 year. Two different parameters were used to evaluate the outcome and finally correlation between the two parameters was evaluated.

\subsection{General observation}

Out of 15 patients only 2 had bilateral intra-articular calcaneal fractures in our series. According to Cave ${ }^{[4]}$, $10 \%$ of the calcaneal fractures are associated with compression fractures of the dorsal or lumbar spine and $25 \%$ are associated with other injuries of lower extremity. None of the patients had other fractures with calcaneal fractures.

Table 3: Comparison between present study and previous ones

\begin{tabular}{|c|c|c|c|c|c|c|}
\hline Series & Year & Cases & Excellent & Good & Fair & Poor \\
\hline Rowe & 1963 & 31 & $12.9 \%$ & $51.6 \%$ & $29.0 \%$ & $6.5 \%$ \\
\hline Sanders & 1992 & 120 & $25 \%$ & $40.8 \%$ & $10.8 \%$ & $23.3 \%$ \\
\hline Zwipp & 1992 & 90 & $16.4 \%$ & $44.7 \%$ & $32.4 \%$ & $6.5 \%$ \\
\hline Richard & 2002 & 20 & $37.5 \%$ & $25 \%$ & $12.5 \%$ & $25 \%$ \\
\hline Our series & 2016 & 17 & $23.52 \%$ & $41.17 \%$ & $23.52 \%$ & $11.76 \%$ \\
\hline
\end{tabular}

The outcome of surgically managed cases is comparable to the results got in other studies (Rowe, Sanders and Zwipp ${ }^{[10]}$ ). But the size of our study group is too small to make a conclusion.

Our study shows that restoration of the Bohler's angle is possible with operative management. Similar study was done by D Makki et al. ${ }^{[9]}$ in 2010. They carried out a retrospective review of 47 intra-articular fractures of the calcaneum treated by open reduction and internal fixation in 45 patients by a single surgeon. They concluded that Restoration of Bohler's angle was associated with a better outcome and that prompt osteosynthesis should be considered for intra-articular fractures of the calcaneum in order to restore the shape of the hindfoot and Bohler's angle.
Our study shows a good correlation between post treatment Bohler's angle and Rowe score in operative group. This indicated that with the restoration of Bohler's angle, the functional outcome is much better. Similar study was done by Paul et al. ${ }^{[11]}$ in 2004. They assessed the long-term results after operative and non-operative treatment of undisplaced and displaced calcaneal fractures. They reviewed 70 patients at an average period of 6.5 years. They concluded that patients with undisplaced calcaneal fractures had a good outcome. Those with displaced fractures treated surgically who presented at follow-up with a BA $>10^{\circ}$ had a satisfactory functional outcome and those with displaced fractures who had non-operative treatment had a poor outcome. The poorest outcome was consistently seen in patients who were treated operatively without restoration of BA. They also concluded that BA has a prognostic importance.

The mean post-operative Gissanes angle in our study was $132.35^{\circ}$. Long $\mathrm{C}$ et al. ${ }^{[12]}$ concluded that the mean postoperative Bohler's angle and Gissane's angle were $25.31^{\circ}$ and $117.5^{\circ}$ respectively, and the overall excellent to good rate was as high as $82.6 \%$, which was related to normal Bohler's angle and Gissane's angle regained by operation. Thus, it confirmed that the recovered Bohler's angle and Gissane's angle were important for functional recovery of elderly patients with calcaneal fracture.

It was also noted that the patients with one of the listed comorbidities were more prone to develop a complication post operatively. It has previously been proved in multiple trials that the presence of a smoking habit commonly leads to a wound complication post operatively due to the effect on the microvasculature. The same holds true regarding the presence of Diabetes in these patients. It is, however, interesting to note that, a study conducted by Zhang et al. ${ }^{[8]}$, found that smoking, hypertension, and drinking did not make significant differences in the risk of wound complications. Diabetes was the strongest risk factor for wound complications. Patients with diabetes had a 9.97-times greater risk of wound complications when compared with patients without diabetes $^{8}$. We also found that the functional outcomes were significantly decreased in those patients who developed complications during the study.

The most common patient complaints were pain. Four fractures $(23.52 \%)$ developed pain in our study. There could be many factors causing pain. Subtalar incongruity, or penetration of implants into the subtalar joint, or arthritis may lead to pain. We can judge the pain through different parts of the foot. Lateral pain is caused by lateral impingement and peroneal tendon pathology, which is characterized by resisted eversion aggravating pain. Anterior pain results primarily from either talar neck impingement or scar tissue in the ankle. Plantar pain secondary to plantar exostosis is the most common, while poorly localized pain is caused by nerverelated problems or complex regional pain syndrome ${ }^{[13]}$.

Hence, our analysis confirms that the most frequent long-term result of a calcaneal fracture is the persistence of disability and pain for many years after the trauma, but not enough to significantly affect the activities of daily life.

Wound necrosis was one of the common complications noted in the patient group who underwent an extended lateral incision and ORIF with plating. In our study, $17.6 \%$ (out of 17 cases) developed wound necrosis. Wound infection is the common complication in some previous studies about operative fixation of calcaneal fractures. Svatoš et al. ${ }^{[14]}$ reported deep wound infection rate of $3.7 \%$. Court-Brown ${ }^{[15]}$ reported that there were $10(5.6 \%)$ deep infections and 35 
$(19.7 \%)$ superficial infections. Wallace ${ }^{[16]}$ pointed that the management goal for these infections was to prevent direct extension to bone causing osteomyelitis. So radical debridement and aggressive antimicrobial therapy are mandatory in cases of infections, but the plate and screws should be removed if infection is because of lack of effective control. In our study, infections of 4 fractures were cured with debridement and aggressive antimicrobial therapy. At the same time we think that soft tissue coverage with local or free flaps has to be considered to avoid protracted courses, shorten operation time and delayed time to surgery can reduce the risk of infection.

Wound necrosis is the most frequently observed postoperative complication in calcaneal fractures, which is seen in up to $14 \%$ of cases after standard osteosynthesis via an extended lateral approach and up to $27 \%$ with a bilateral approach. To avoid wound necrosis, time to surgery should be delayed until wrinkling of the skin reappears, and the leg must be elevated and cold compresses started on admission in our experience.

In our study there was an incidence of $23.5 \%$ of open fractures and $76.5 \%$ of closed fractures. Schepers et al. ${ }^{[17]}$ revealed in his study, an incidence of $93 \%$ closed and $7 \%$ of open fractures. The open wound was commonly found on the medial side.

Closed fractures were found to have a significantly improved functional outcome than open fractures. This was due to the need for aggressive management of the open wounds of these fractures, due to high incidence of wound complications in these patients. In our study, there were 4 patients with open fractures, 3 of which had developed a wound complication post operatively. Also there was a factor of increased hospital stay and the management of these injuries should include intravenous antibiotics, tetanus prophylaxis, and urgent debridement and irrigation. Patients were found to have higher chances of complications and unsatisfactory outcomes. In our study, patients with open fractures presented at the end follow up visit with persistent pain. This could be attributed to the initial soft tissue disruption leading to possible chronic heel pad pain. All patients with open fractures in our study were found to have complications.

In our study, we also observed that in some cases, also nonoptimal results were judged satisfactory with the Rowe score if the patient was conscious of the seriousness of his trauma and of the impossibility to eliminate the consequences of it. On the other hand, only $23.5 \%$ of subjects enrolled were completely satisfied.

As per the Rowe's Questionnaire ${ }^{12}$, patients who had worked before their trauma resumed work in $82.3 \%$ of cases, although they have had some level of restriction on returning to their previous line of work. De Groot et al. also reported $80 \%$ of ORIF patients resuming their work. $23.52 \%$ patients had variable amounts of stiffness and thereby difficulty in walking on uneven surfaces and running.

Table 4: Modified Rowe Score for Clinical Evaluation

\begin{tabular}{|c|c|c|}
\hline \multirow{5}{*}{ Pain } & None & 30 \\
\hline & Exercise induced & 25 \\
\hline & Mild on daily activities & 20 \\
\hline & Pain with weight bearing & 10 \\
\hline & Pain at rest & 0 \\
\hline \multirow{4}{*}{$\begin{array}{c}\text { Range of moton } \\
(\%)\end{array}$} & 100 to 75 & 20 \\
\hline & 74 to 50 & 10 \\
\hline & 49 to 25 & 5 \\
\hline & 25 to 0 & 0 \\
\hline \multirow{4}{*}{ Gait } & Normal & 15 \\
\hline & Mild limp (excersice) & 10 \\
\hline & Moderate limp & 5 \\
\hline & Severe limp & 0 \\
\hline \multirow{5}{*}{ Activities } & Normal & 20 \\
\hline & Restricted on rough ground & 15 \\
\hline & Moderate daily restrictions & 10 \\
\hline & Able to walk short distances only & 5 \\
\hline & Unable to walk & 0 \\
\hline \multirow{4}{*}{ Work } & No restrictions & 15 \\
\hline & Some restriction on usual occupation & 10 \\
\hline & Change of job or substantial restriction & 5 \\
\hline & Unable to work & 0 \\
\hline Total & & 100 \\
\hline
\end{tabular}

- Method of measurement of outcome of interest - Modified Rowe Score ${ }^{12}$

100-90: Excellent 89-75: Good

$74-51$ : Fair 50 or less: Poor

\section{Conclusions}

- The outcome of surgically managed cases is comparable to the outcomes in other studies. But the size of the present study group is too small to make an emphatic conclusion; although it adds to the pool of knowledge data.

- In case of displaced and comminuted calcaneal fractures the aim of the treatment should be to achieve reduction and restoration of Bohler's angle. Surgery perhaps is the solution to achieve that goal.
- Post-operative Bohler's angle has a prognostic importance on functional outcome.

- Significant number of complications are associated with these fractures which are seen both with conservative and operative management. The incidence is more in open fractures as compared to closed fractures, as was found in our study as well.

- In our study also open calcaneal fractures have poor outcome, with risk of wound infection and delayed wound healing, which is comparable to other studies. 
- In closed displaced fractures, where surgery was done, the functional results were better when restoration of Bohler's angle was achieved to an angle above $23^{\circ}$.

- The comparison between radiological measurements and clinical outcomes showed that the radiographic classification systems of Essex-Lopresti and Sanders are both useful and reliable prognostic tools to guide clinical decision in the treatment of these challenging fractures.

- In our study the average period of Follow up is 1 year and long term problems of early degenerative arthritis is not taken in to consideration, hence adding to the limitations in our study.

- However, as reported in the literature, our results confirm that operative treatment is characterized by a high rate of complications, mainly related to surgical wound dehiscence and infections.

- Hence, we strongly believe that ORIF has to be planned only when swelling and fracture blisters are completely subsided.

\section{References}

1. Bankart Massart P, Delvaux D. The operative treatment of intraarticular calcaneal fractures: indications, technique and results in 257 cases. Clin Orthop. 1993; 290:55-58.

2. Conn HR. The treatment of fractures of the os calcis. $\mathbf{J}$ Bone Joint Surg. 1935; 17:392-405.

3. Bellabarba C, Sangeorzan BJ. Fractures of the calcaneus. Orthop Clin North Am. 1999; 33:263-266.

4. Koval K, Sanders R. the radiologic evaluation of calcaneal fractures. Clin Ortho. 1993; 290:41-58.

5. Mahajan BK. Methods in biostatistics for medical students and research workers. 5th ed: New Delhi: Jaypee Brothers, 1997.

6. Yeo et al. BMC Musculoskeletal Disorders. 2015; 16:63.

7. Su et al. BMC Surgery. 2013; 13:40. http:// www. Biomedcentral. Com /1471-2482/13/40

8. Zhang et al. Scandinavian Journal of Trauma, Resuscitation and Emergency Medicine. 2015; 23:18.

9. Makki D, Alnajjar HM, Walkay S, Ramkumar U. Osteosynthesis of displaced intra-articular. J Bone Joint Surg. 2010; 92:693-700.

10. Zwipp H, Tscherne H, Thermann $\mathrm{H}$ et al. Osteosynthesis of displaced intraarticular fractures of the calcaneus: results in 123 cases. Clin Orthop. 1993; 290:76-86.

11. Paul M, Peter R, Hofmayer P. Fractures of the calcaneus: a review of 70 patients. J Bone Joint Surg. 2004; 86B:1142-1156.

12. Burdeaux BD. Reduction of calcaneal fractures by the McRaynolds medial approach and its experimental basis. CIin Orthop. 1983; 177:87-90.

13. Paley D, Fischgrund J. Open reduction and circular external fixation of intraarticular calcaneal fractures. Clin Orthop. 1993; 290:125-140.

14. Paul M, Peter R, Hofmayer P. Fractures of the calcaneus: a review of 70 patients. J Bone Joint Surg. 2004; 86B:1142-1156.

15. Gould N. Lateral approach to the os calcis. Foot Ankle. 1984; 4:212-220.

16. Buckley R, Tough S, McCormack. Operative compared with non-operative treatment of displaced intraarticular calcaneal fractures. J Bone Joint Surg. 2002; 84A:17331740 .

17. Schepers $\mathrm{T}$ et al., Percutaneous calcaneal fracture surgery, J Orthop Sci. 2007; 12:22-27. 